\section{Kolonkarzinom: Längere Therapie ist nicht besser}

\author{
Dass Patienten mit Kolonkarzinom in einem höheren Stadium von einer \\ adjuvanten Chemotherapie profitieren, ist unstrittig. Aber wie lange sollte \\ die Therapie dauern? Japanische Kollegen gingen dieser Frage im Rahmen \\ einer randomisierten Studie nach.
}

P atienten mit höhergradigem Kolonkarzinom erhalten üblicherweise eine adjuvante Chemotherapie. Wie lang diese aber dauern sollte, wird kontrovers diskutiert. In Japan wird die Kombination aus Uracil und Tegafur/ Folinsäure weithin als Standard angesehen. Deshalb haben japanische Kollegen mit dieser Therapie als Grundlage eine Phase-III-Studie durchgeführt, in der sie untersuchen wollten, ob Patienten mit Kolonkarzinom im Stadium IIB/III von einer besonders langen Behandlung profitieren.

In die Studie wurden 1.071 Patienten aus 233 verschiedenen Kliniken aufgenommen, deren Tumor sich kurativ resezieren ließ. Sie wurden binnen 6 Wochen nach der Operation randomisiert.
Die Kurzzeit-Gruppe erhielt Tegafur/Folinsäure für jeweils 28 Tage eines 35-Tage-Zyklus über 6 Monate. Die LangzeitGruppe bekam Tegafur/Folinsäure an 5 konsekutiven Tagen pro Woche über 18 Monate. Primärer Endpunkt war das krankheitsfreie Überleben (DFS), sekundäre Endpunkte waren das Gesamtüberleben und die Sicherheit der Behandlung.

Ein statistisch signifikanter Unterschied im DFS wurde zwischen den beiden Gruppen nicht festgestellt. Nach 5 Jahren betrug die DFS-Rate $69 \%$ in der Langzeit-Gruppe und $69 \%$ im KurzzeitArm. Die 5-Jahres-Gesamtüberlebensrate lag bei $85 \%$ im Langzeit-Arm und ebenfalls bei $85 \%$ in der KurzzeitGruppe.

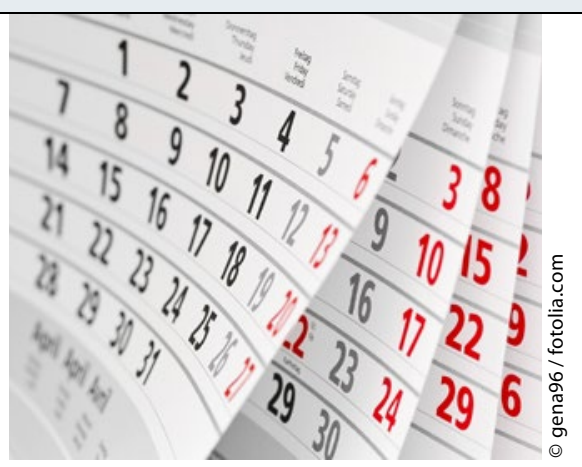

Eine dreimal so lange adjuvante Therapie ist nicht von Vorteil.

Fazit: Die adjuvante Behandlung von Patienten mit höhergradigem Kolonkarzinom über 18 Monate mit Tegafur/ Folinsäure verbessert im Vergleich zur 6-monatigen Therapie das DFS und das Gesamtüberleben nicht. Eine Behandlungsdauer von 6 Monaten scheint für die adjuvante Chemotherapie des Kolonkarzinoms im Stadium IIB/III ausreichend zu sein.

Christina Berndt

Sadahiro S et al. Randomized phase III trial of treatment duration for oral uracil and tegafur plus leucovorin as adjuvant chemotherapy for patients with stage IIB/III colon cancer: final results of JFMC33-0502. Ann Oncol. 2015; 26(11):2274-80.

\title{
XELOX bei Kolonkarzinomen besteht den Langzeitcheck
}

Die adjuvante Therapie des Kolonkarzinoms im Stadium III mit Oxaliplatin plus Capecitabin (XELOX) verlängert den primären Ergebnissen einer Phase-III-Studie zufolge das krankheitsfreie Überleben (DFS) gegenüber der Bolus-Therapie mit Fluorouracil/Folinsäure (FU/FA). Jetzt liegen finale Daten zum Gesamtüberleben vor.

n der Studie hatten 1.866 Patienten nach kurativer Resektion eines Kolonkarzinoms (Stadium III) randomisiert entweder Oxaliplatin $\left(130 \mathrm{mg} / \mathrm{m}^{2}\right.$ an Tag 1) und Capecitabin $\left(1.000 \mathrm{mg} / \mathrm{m}^{2}\right.$ bid an den Tagen 1-14 alle 3 Wochen; $\mathrm{n}=944$ ) oder eine Bolustherapie mit FU/FA über 6 Monate nach dem Mayo-Clinic- oder Roswell-Park-Regime erhalten ( $\mathrm{n}=942)$. Der primäre Endpunkt, das DFS nach 3 Jahren, zeigte einen klaren Vorteil für XELOX mit einer Hazard Ratio (HR) von 0,80 (95\%-Konfidenzintervall [95\%-KI] $0,69-0,93 ; \mathrm{p}=0,0045)$.

Die jetzt ausgewertete 7-Jahres-Rate krankheitsfrei überlebender Patienten lag bei $63 \%$ unter XELOX und $56 \%$ un- ter FU/FA (HR 0,80, 95\%-KI 0,69-0,93; $p=0,004$ ), wobei der Vorteil für XELOX nach jedem Jahr konsistent war. Der früh gezeigte DFS-Vorteil übersetzte sich auch in einen Vorteil hinsichtlich des 7-Jahres-Gesamtüberlebens (OS; $73 \%$ unter XELOX vs. $67 \%$ unter FU/FA; HR 0,83, $95 \%$-KI 0,70-0,99; $\mathrm{p}=0,04$ ).

In die Studie war auch eine Biomarkeranalyse bei 498 Patienten integriert. Die Auswertungen deuteten in der XELOXGruppe darauf hin, dass die Expression der Dihydropyrimidin-Dehydrogenase (DPD) der Tumorzellen prädiktiv sein könnte für die Wirksamkeit dieser Therapie: Die HR für das DFS lag beim Vergleich von hohen zu niedrigen DPD-Ra- ten bei 2,45 (95\%-KI 1,55-3,86; $\mathrm{p}<0,001)$. Ein entsprechende Ergebnis zeigte sich auch für das OS (HR 2,75, $95 \%$-KI 1,654,59; $\mathrm{p}<0,001)$. In der FU/FA-Gruppe ließ sich keine Assoziation von Wirksamkeit und DPD-Expressionslevel oder einem anderen Biomarker feststellen.

Fazit: Über median 7 Jahre verbesserte XELOX bei Patienten mit reseziertem Kolonkarzinom im Stadium III das OS gegenüber einer FU/FA-Bolustherapie und kann damit als Standard in dieser Situation gelten. Die DPD-Expression könnte ein vielversprechender prädiktiver und klinisch relevanter Biomarker für die Wirksamkeit von XELOX sein, was in weiteren Studien prospektiv untersucht werden sollte. Friederike Klein

Schmoll HJ et al. Capecitabine Plus Oxaliplatin Compared With Fluorouracil/Folinic Acid As Adjuvant Therapy for Stage III Colon Cancer: Final Results of the NO16968 Randomized Controlled Phase III Trial. J Clin Oncol. 2015;33(32):3733-40. 